\title{
Mise en œuvre d'une réforme en Suisse
}

Jean Racine

\section{(2) OpenEdition}

\section{Journals}

Édition électronique

URL : http://journals.openedition.org/ries/3395

DOI : $10.4000 /$ ries.3395

ISSN : 2261-4265

\section{Éditeur}

Centre international d'études pédagogiques

\section{Édition imprimée}

Date de publication : 1 mars 1996

Pagination : 91-98

ISSN : 1254-4590

\section{Référence électronique}

Jean Racine, « Mise en œuvre d'une réforme en Suisse », Revue internationale d'éducation de Sèvres [En ligne], 09 | 1996, mis en ligne le 19 août 2013, consulté le 23 mars 2021. URL : http:// journals.openedition.org/ries/3395; DOI : https://doi.org/10.4000/ries.3395

Ce document a été généré automatiquement le 23 mars 2021.

(c) Tous droits réservés 


\title{
Mise en œuvre d'une réforme en Suisse
}

\author{
Jean Racine
}

1 C'est en 1975 que la Conférence suisse des directeurs cantonaux de l'Instruction publique (CDIP) ${ }^{1}$ a recommandé aux vingt-six cantons et demi-cantons une réforme de l'enseignement d'une deuxième langue nationale. Il faut insister sur le fait qu'il ne s'agissait là que de recommandations, ce qui leur laissait, ainsi qu'aux régions, la possibilité d'une mise en œuvre à leur gré et au rythme qui leur semblait le plus approprié.

2 Ces recommandations prévoyaient une réforme sur le plan des structures et des contenus ainsi que sur le plan plus général de l'enseignement ${ }^{2}$.

\section{Les structures}

3 En ce qui concerne les structures, la CDIP a retenu les principes suivants pour l'enseignement de la langue 2 qui devra être obligatoire pour tous les élèves; débuter selon le système scolaire des cantons, en $4^{\mathrm{e}}$ ou $5^{\mathrm{e}}$ année scolaire ${ }^{3}$; être prodigué par l'enseignant principal (maître généraliste); de plus, cet enseignement ne devra pas avoir au niveau du primaire d'effet ni sur les critères de promotion et de sélection ni sur le passage dans le secondaire ${ }^{4}$; la langue 2 sera le français pour la Suisse alémanique, l'allemand pour la Suisse romande, le français et l'allemand pour le $\operatorname{Tessin}^{5}$; enfin, l'enseignement de la langue 2 subira une profonde réforme.

4 Les recommandations de 1975 seront réalisées en l'an 2000 dans tous les cantons suisses (sauf dans le canton d'Argovie). 
La Suisse des langues

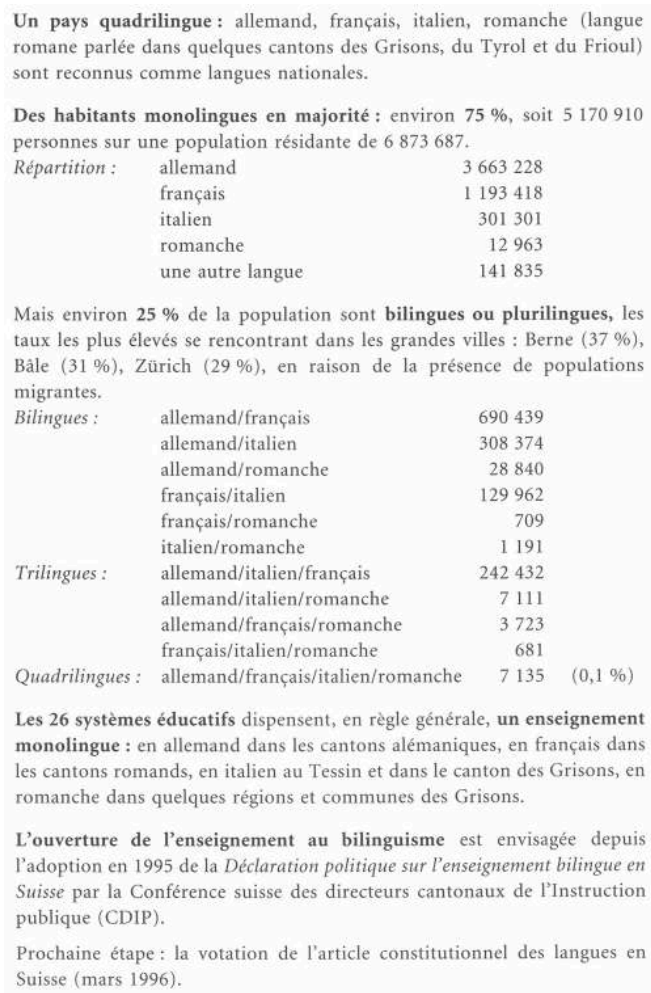

Mais environ $25 \%$ de la population sont bilingues ou plurilingues, les taux les plus élevés se rencontrant dans les grandes villes : Berne ( $37 \%$ ), Bâle $(31 \%)$, Zürich $(29 \%)$, en raison de la présence de populations migrantes.

Bilingues :

allemand/français $\quad 690439$ allemand/italien $\quad 308374$ allemand/romanche $\quad 28840$ français/italien $\quad 129962$ français/romanche $\quad 709$ italien/romanche $\quad 1191$

Trilingues: $\quad \begin{array}{ll}\text { italien/romanche } & 1191 \\ \text { allemand/italien/français } & 242432\end{array}$ $\begin{array}{ll}\text { allemand/italien/romanche } & 7111 \\ \text { allemand/français/romanche } & 3723\end{array}$ allemand/français/romanche $\quad 3723$ Quadrilingues: allemand/français/italien/romanche $7135 \quad(0,1 \%)$

Les 26 systèmes éducatifs dispensent, en règle générale, un enseignement monolingue : en allemand dans les cantons alémaniques, en français dan les cantons romands, en italien au Tessin et dans le canton des Grisons, en romanche dans quelques régions et communes des Grisons.

L'ouverture de l'enseignement au bilinguisme est envisagée depuis l'adoption en 1995 de la Déclaration politique sur l'enseignement bilingue en Suisse par la Conférence suisse des directeurs cantonaux de l'Instruction publique (CDIP).

Prochaine étape: la votation de l'article constitutionnel des langues en Suisse (mars 1996).

\section{Les contenus}

5 Sur le plan des contenus, la réforme consiste en une réforme interne de l'enseignement des langues : elle concerne aussi bien des aspects didactiques que le passage d'un degré scolaire à un autre, le perfectionnement des enseignants ainsi que les moyens d'enseignement.

\section{Aspects didactiques et méthodologiques}

\section{Les objectifs}

6 Les objectifs retenus se fondent sur les savoir-faire à atteindre. Les connaissances du système formel de la langue sont fonctionnalisées dans la perspective d'un enseignement à des fins de communication qui permette aux élèves d'acquérir une langue pour s'en servir avec des interlocuteurs la pratiquant comme langue première.

7 L'enseignement/apprentissage sera axé sur les quatre compétences linguistiques selon les répartitions suivantes : 


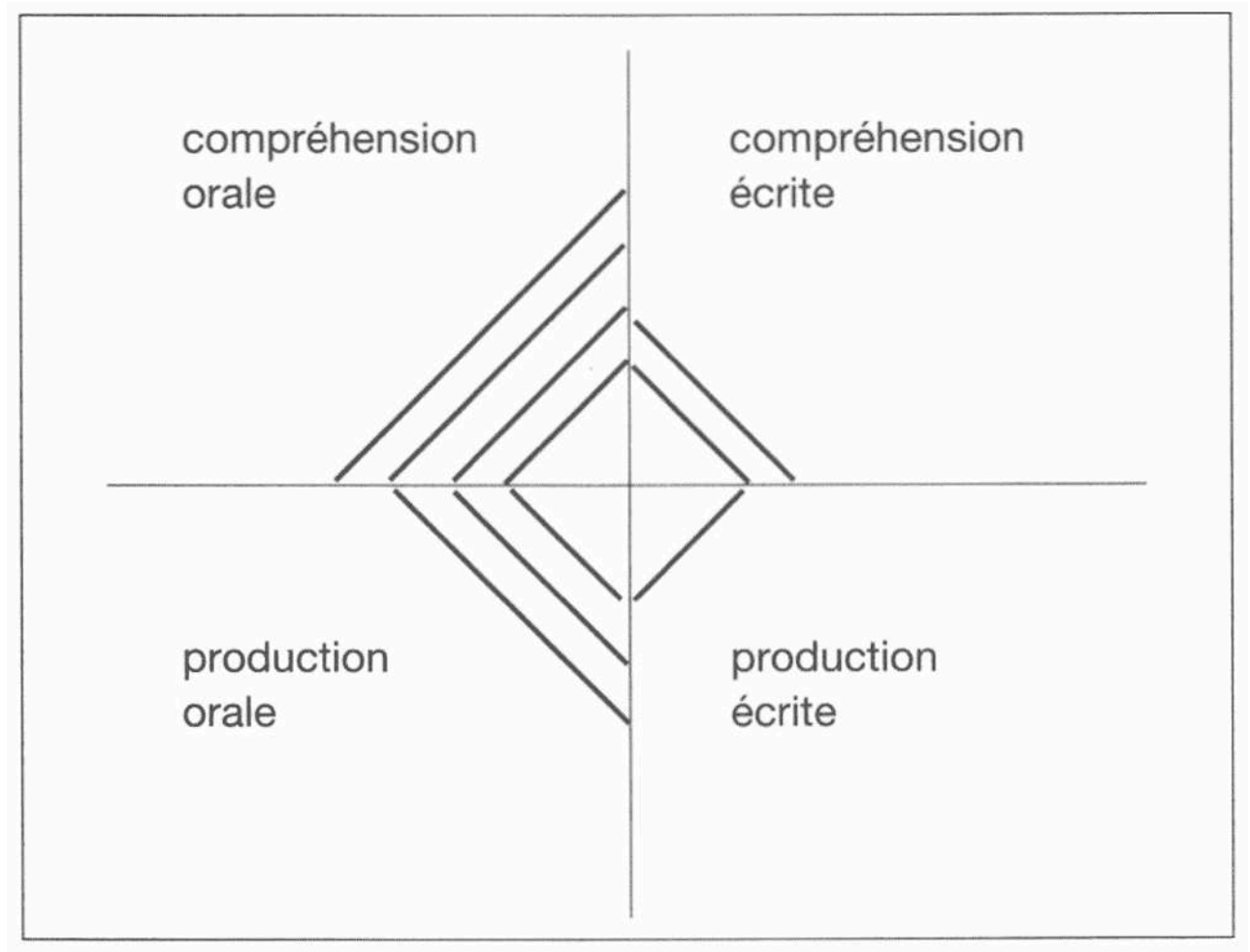

\section{Attitudes des élèves vis-à-vis du multiculturalisme}

8 Le contact avec d'autres personnes permet d'élargir l'éventail des expériences des élèves et de leur faire comprendre que les différences culturelles et linguistiques ne sont pas contraignantes mais enrichissantes. L'enseignement apprentissage d'une deuxième langue nationale doit favoriser des contacts avec des interlocuteurs parlant une autre langue que la leur. Ainsi l'enseignement de la deuxième langue prend une dimension interculturelle permettant aux élèves d'acquérir une culture de l'altérité.

\section{Aptitudes à la communication}

9 Les élèves doivent acquérir les aptitudes nécessaires à la communication, ils doivent être capables de comprendre et de se faire comprendre, ils doivent apprendre à formuler leurs besoins et à exprimer leurs opinions. Pour cela, l'enseignement utilisera méthodiquement des techniques d'apprentissage et permettra aux élèves de développer leur responsabilité et leur autonomie dans les processus d'apprentissage.

\section{Développement des compétences}

10 L'importance accrue attribuée à l'expression orale a entraîné un déplacement des priorités, un changement dans la manière d'enseigner et la nécessité de mettre en place d'autres formes d'évaluation.

11 Un accent particulier est mis également sur le développement des compétences de compréhension de messages parlés et de textes écrits, ce qui impliquera des répercussions du point de vue de la réalisation de nouveaux matériaux d'apprentissage, l'emploi de textes authentiques et l'intégration, dans le processus d'apprentissage, de nouvelles stratégies de compréhension. 


\section{Coordination verticale} à prendre place dans la formation initiale des maitres. Dans plusieurs cantons, la didactique de la langue 2 a été ajoutée aux programmes. Des stages pour la formation linguistique ont été mis en place. Cependant, il faudra encore fournir de grands efforts jusqu'à ce que les plans "d'études-cadres " ${ }^{6}$ élaborés en 1982 soient définitivement institutionnalisés dans tous les cantons.

Pour ce qui est de la formation des enseignants dans le secondaire 2, elle se fait en règle générale dans les différentes universités. Elle manque cependant d'une coordination systématique. La formation initiale pour les enseignants du secondaire 1 et du secondaire 2 est réglementée dans les différents cantons. D’une manière générale, on peut dire que les enseignants du secondaire sont obligés d'effectuer des stages d'une durée de trois à neuf mois dans une région ou un pays où la langue à apprendre est langue 1. 


\section{La formation continue} promouvoir, à l'intérieur du pays, les apprentissages des autres langues nationales. Il est clair que le choix du lieu, qui n'est pas fixé d'avance par des mesures cantonales, reste en rapport avec des représentations "stéréotypes " dont les enseignants sont imprégnés. Les Alémaniques ont en général une attitude positive envers la Suisse romande. La langue française jouit chez eux d'un prestige considérable. Par contre, les représentations que les Romands ont des Alémaniques s'avèrent moins positives: méfiance par rapport à la majorité politique, refus des dialectes parlés en Suisse alémanique, consternation devant le fait qu'en Suisse alémanique (si l'on réduit la situation linguistique à une formule un peu simple) on lise et écrive en allemand standard («le bon allemand » comme disent les Romands) mais que l'on s'entretienne dans l'un des dialectes alémaniques qui ne sont pas enseignés systématiquement dans les écoles romandes. Tout cela peut expliquer que la formation linguistique en allemand pour les enseignants du primaire romand, ait lieu en dehors de notre pays. Ceci s'avère d'autant plus paradoxal que les recommandations de la CDIP de 1975 et de 1986 mettaient en avant l'amélioration des contacts et de la compréhension entre les régions linguistiques. La diglossie " dialectes alémanique-allemand standard " pose en Suisse alémanique des problèmes évidents mais il faut ajouter le fait que depuis l'abolition du mur de Berlin et l'ouverture vers l'Allemagne et les pays de l'Est, l'allemand standard a connu un nouvel essor en Europe. Cela peut aussi expliquer que les enseignants romands aient plutôt tendance à aller apprendre l'allemand en Allemagne plutôt qu'en Suisse alémanique.

21 Dans les cours de formation continue, la didactique occupe, selon les cantons, pour les enseignants du primaire, un temps variant entre une à quatre semaines. Tous les programmes de ces cours sont en rapport avec les objectifs préconisés par la CDIP en 1975 et en 1986.

22 Une difficulté importante réside - pour l'articulation primaire/secondaire déjà évoquée- en un certain traditionnalisme didactique des enseignants du secondaire 1 et 2. Pour eux, la formation didactique en langue 2 n'est pour ainsi dire nulle part obligatoire: elle n'atteindra que les enseignants intéressés par des questions de réforme et qui ne sont pas hostiles à leur principe. 


\section{La création de nouveaux moyens d'enseignement} engues, les procédés d'enseignement/apprentissage peuvent et doivent être développés en vue d'une plus grande efficacité, nécessitée par la construction de l'Europe. C'est dans cette optique qu'il faut situer la dernière déclaration de la CDIP concernant la "Promotion de l'enseignement bilingue en Suisse » qui a été adoptée en 1995 (voir document annexe).

cette déclaration, la CDIP affirme sa volonté de s'engager. Ce sont les cantons qui devront relever le défi et trouver les concrétisations au niveau scolaire susceptibles de rendre plus efficaces encore l'enseignement des langues vivantes en Suisse. 


\section{Déclaration de la CDIP et des directeurs cantonaux de l'économie publique responsables de la formation professionnelle} disciplines non linguistiques, précédé, accompagné ou suivi d'un enseignement de la langue étrangère elle-même - est un moyen approprié pour accroître l'efficacité de l'apprentissage des langues et compléter d'autres formes d'enseignement des langues étrangères :

- l'enseignement bilingue devrait être offert dans tous les types d'écoles, en tenant compte de l'âge des apprenants et de la situation locale ;

- il faut permettre et encourager l'obtention de certificats de maturité portant la mention " enseignement bilingue»;

- les autorités sont appelées à éliminer tout ce qui, du point de vue juridique et administratif, pourrait faire obstacle à l'introduction d'un enseignement bilingue et à créer, au niveau de la formation des enseignants, du matériel d'apprentissage et du matériel d'enseignement, des conditions favorables à cet enseignement.

La CDIP s'engage à promouvoir l'échange d'informations et d'expériences sur les projets à venir, les modèles existants, les résultats de la recherche et les évaluations effectuées dans le domaine de l'enseignement bilingue. Cela devrait permettre d'éviter des doubles emplois et d'assurer une coordination optimale des différents travaux. 


\section{NOTES}

1. Les directeurs ou directrices de l'enseignement public sont, en d'autres termes, les ministres de l'Éducation des cantons.

2. Je résume les résultats d'une enquête systématique entreprise par la Commission langue 2 de la CDIP CH et publiée dans Dossier 20 CDIP, « Réforme de l'enseignement de la langue seconde dans les écoles obligatoires », Berne, CDIP, 1992.

3. Dans le canton du Tessin, l'enseignement du français commence à la $3^{\mathrm{e}}$ année de la scolarité, celui de l'allemand à la $7^{\mathrm{e}}$ année.

4. Les cantons bilingues (Berne, Valais, Fribourg) ainsi que le canton de Bâle-Ville n'ont pas tenu compte de cette recommandation qui avait été retenue pour garantir un enseignement ludique et sans contrainte de notation (ce qui n'exclut pas l'évaluation) au niveau primaire.

5. Le canton d'Uri a décidé, en se basant sur les recommandations qui proposaient - en accord avec les propositions du Conseil de l'Europe - que la langue 2 soit la langue du voisin, d'enseigner l'italien. Il est probable que le canton des Grisons adoptera dans les parties non romanches la même démarche, étant donné la proximité et le prestige de l'italien dans les Grisons.

6. Plans "d'études-cadres" pour la formation des instituteurs en vue de l'enseignement du français (langue 2) pendant la scolarité obligatoire, Berne, CDIP, 1992.

7. Beat Vonarburg, Jean Racine, Joseph Lischer, Funkkolleg Französisch/ Cours de français, Lucerne, ILZ, 1983.

8. Bulletin langue 2 (1-1995 et 1-1996), Berne, CDIP, 1995 et 1996.

\section{RÉSUMÉS}

Les recommandations de 1975 de la Conférence suisse des directeurs cantonaux de l'Instruction publique (CDIP) concernant l'enseignement d'une langue 2 a permis une évolution très positive tant sur les contenus et sur les moyens didactiques de cet enseignement que sur le perfectionnement et les moyens pédagogiques utilisés. La Déclaration sur «la promotion de l'enseignement bilingue », adoptée en 1995, conforte et encourage cette politique.

\section{INDEX}

Index géographique : Suisse

Mots-clés : école primaire, enseignement des langues, langues étrangères, réforme, enseignement précoce des langues vivantes, enseignement d'une langue seconde

\section{AUTEUR}

\section{JEAN RACINE}

Président de la Commission langues vivantes de la CDIP Nord-Ouest, canton de Soleure, Suisse. 